\title{
KATRIN bound on $3+1$ active-sterile neutrino mixing and the reactor antineutrino anomaly
}

\author{
C. Giunti, ${ }^{a}$ Y.F. Li ${ }^{b, c}$ and Y.Y. Zhang ${ }^{b, c}$ \\ ${ }^{a}$ Istituto Nazionale di Fisica Nucleare (INFN), Sezione di Torino, \\ Via P. Giuria 1, I-10125 Torino, Italy \\ ${ }^{b}$ Institute of High Energy Physics, Chinese Academy of Sciences, \\ Beijing 100049, China \\ ${ }^{c}$ School of Physical Sciences, University of Chinese Academy of Sciences, \\ Beijing 100049, China \\ E-mail: carlo.giunti@to.infn.it, liyufeng@ihep.ac.cn, \\ zhangyiyu@ihep.ac.cn
}

ABSTRACT: We present the bounds on $3+1$ active-sterile neutrino mixing obtained from the first results of the KATRIN experiment. We show that the KATRIN data extend the Mainz and Troitsk bound to smaller values of $\Delta m_{41}^{2}$ for large mixing and improves the exclusion of the large- $\Delta m_{41}^{2}$ solution of the Huber-Muller reactor antineutrino anomaly. We also show that the combined bound of the Mainz, Troitsk, and KATRIN tritium experiments and the Bugey-3, NEOS, PROSPECT, and DANSS reactor spectral ratio measurements exclude most of the region in the $\left(\sin ^{2} 2 \vartheta_{e e}, \Delta m_{41}^{2}\right)$ plane allowed by the Huber-Muller reactor antineutrino anomaly. Considering two new calculations of the reactor antineutrino fluxes, we show that one, that predicts a lower ${ }^{235} \mathrm{U}$ antineutrino flux, is in agreement with the tritium and reactor spectral ratio measurements, whereas the other leads to a larger tension than the Huber-Muller prediction. We also show that the combined reactor spectral ratio and tritium measurements disfavor the Neutrino-4 indication of large active-sterile mixing. We finally discuss the constraints on the gallium neutrino anomaly.

Keywords: Neutrino Physics, Beyond Standard Model

ARXIV EPRINT: 1912.12956 


\section{Contents}

1 Introduction 1

2 Three neutrino mixing 2

$\begin{array}{lll}3 & 3+1 & \text { sterile neutrino mixing }\end{array}$

$\begin{array}{lll}4 & \text { The reactor antineutrino anomaly } & 7\end{array}$

5 Neutrino-4 11

$6 \quad$ The gallium neutrino anomaly 12

$\begin{array}{lll}7 & \text { Conclusions } & 14\end{array}$

\section{Introduction}

The KATRIN collaboration presented recently [1] the first results of their high-precision measurement of the electron spectrum from ${ }^{3} \mathrm{H}$ decay near the end point, where it is sensitive to neutrino masses at the $\mathrm{eV}$ level. They obtained an upper limit of $1.1 \mathrm{eV}$ at $90 \%$ confidence level (CL) for the effective neutrino mass

$$
m_{\beta}=\sqrt{\sum_{k=1}^{3}\left|U_{e k}\right|^{2} m_{k}^{2}},
$$

in the standard three-neutrino mixing framework, where $U$ is the mixing matrix and $m_{k}$ is the mass of the neutrino $\nu_{k}$, with $k=1,2,3$.

The KATRIN collaboration measured the electron spectrum down to $Q-35 \mathrm{eV}$, where $Q \simeq 18.57 \mathrm{keV}$ is the $\mathrm{Q}$-value of ${ }^{3} \mathrm{H}$, that corresponds to the end-point of the electron spectrum in the absence of neutrino mass effects. Using this spectral measurement, it is possible to constrain also the mixing with the electron neutrino of heavier non-standard neutrinos with masses smaller than about $35 \mathrm{eV}$. This is interesting in view of the indications in favor of the existence of such non-standard neutrinos given by the reactor antineutrino anomaly and the gallium neutrino anomaly (see the recent reviews in refs. [2-4]). A possible explanation of these anomalies is short-baseline neutrino oscillations due to the existence of a non-standard neutrino with a mass of the order of $1 \mathrm{eV}$ or larger. Since it is well established that there are only three active flavor neutrinos, in the flavor basis the new neutrino must be sterile. This framework is commonly called $3+1$ active-sterile neutrino mixing.

In this paper, we first calculate in section 2 the upper bound on $m_{\beta}$ in the standard framework of three-neutrino mixing, in order to test the validity of our analysis of the 
KATRIN data by comparing the results with those of the KATRIN collaboration. Then, in section 3, we calculate the KATRIN bounds on active-sterile neutrino mixing and we show that they are more stringent than those of the Mainz [5] and Troitsk [6, 7] experiments discussed in ref. [8]. In section 4, we compare the KATRIN bounds with the results of the $3+1$ analysis of the reactor antineutrino anomaly [9] assuming the standard Huber-Muller reactor antineutrino flux prediction $[10,11]$ and the two new predictions of Estienne, Fallot et al. [12] and Hayen, Kostensalo, Severijns, Suhonen [13]. In section 4, we discuss also the bounds of experiments that measured the reactor antineutrino spectrum at different distances. In section 5, we compare the positive results of the Neutrino-4 reactor experiment [14] with the bounds from the tritium experiments and from the other reactor spectral ratio measurements. In section 6 , we discuss the constraints on the gallium neutrino anomaly. We finally summarize the results in section 7 .

\section{Three neutrino mixing}

In this section we present the results of our analysis of the KATRIN data in the standard framework of three-neutrino mixing. This is useful in order to describe the method that we used in the analysis of the KATRIN data and in order to check its validity by comparing the results for $m_{\beta}$ with those obtained by the KATRIN collaboration [1].

We consider the $\beta$-decay of the gaseous molecular tritium source $\mathrm{T}_{2}$ :

$$
\mathrm{T}_{2} \rightarrow{ }^{3} \mathrm{HeT}^{+}+e^{-}+\bar{\nu}_{e}
$$

The differential electron spectrum is given by

$$
\begin{aligned}
R_{\beta}(E)= & \frac{G_{\mathrm{F}}^{2} \cos ^{2} \theta_{\mathrm{C}}}{2 \pi^{3}}|\mathcal{M}|^{2} F(E, Z+1) \\
& \times\left(E+m_{e}\right) \sqrt{\left(E+m_{e}\right)^{2}-m_{e}^{2}} \\
& \times \sum_{i, j}\left|U_{e i}\right|^{2} \zeta_{j} \varepsilon_{j} \sqrt{\varepsilon_{j}^{2}-m_{i}^{2}} \Theta\left(\varepsilon_{j}-m_{i}\right),
\end{aligned}
$$

where $G_{\mathrm{F}}$ is the Fermi constant, $\theta_{\mathrm{C}}$ is the Cabibbo angle, $\mathcal{M}$ is the nuclear matrix element, $m_{e}$ is the electron mass, $E$ is the kinetic energy of the outgoing electron, $F(E, Z+1)$ is the Fermi function describing the Coulomb effect of the electron, and $Z=1$ is the atomic number of the parent nucleus. A fully relativistic description of the Fermi function is given by

$$
F(E, Z)=2(\gamma+1) \frac{e^{\pi y}}{\left(2 p R_{n}\right)^{2(1-\gamma)}} \frac{|\Gamma(\gamma+i y)|^{2}}{\Gamma(2 \gamma+1)^{2}},
$$

where $y=Z \alpha E / p$ and $\gamma=\sqrt{1-\alpha^{2} Z^{2}}$, with the fine-structure constant $\alpha$ and the complex Gamma function $\Gamma(z)$ [15]. The radius of the ${ }^{3} \mathrm{He}^{2+}$ nucleus is $R_{n}=2.8840 \times 10^{-3} / m_{e}$ [16]. In eq. (2.2), $\varepsilon_{j}=E_{0}-E-V_{j}$ is the neutrino energy, with $E_{0}=M_{\mathrm{T}}-M_{3} \mathrm{He}_{e}-m_{e}$, where $M_{\mathrm{T}}$ and $M_{3} \mathrm{He}$ are, respectively, the mass of the initial and final nucleus. In the calculation of the $\beta$-decay electron spectrum $R_{\beta}(E)$, we considered the excitation states of the 
daughter molecular system, which have excitation energies $V_{j}$ and a final-state distribution with probabilities $\zeta_{j}$. These quantities are calculated with the Born-Oppenheimer approximation and can be found in refs. [17, 18].

When the experimental resolution is much larger than the values of neutrino masses, one can define the effective neutrino mass $m_{\beta}$ as in eq. (1.1) and approximate the differential electron spectrum as

$$
\begin{aligned}
R_{\beta}(E) \simeq & \frac{G_{\mathrm{F}}^{2} \cos ^{2} \theta_{\mathrm{C}}}{2 \pi^{3}}|\mathcal{M}|^{2} F(E, Z+1) \\
& \times\left(E+m_{e}\right) \sqrt{\left(E+m_{e}\right)^{2}-m_{e}^{2}} \\
& \times \sum_{j} \zeta_{j} \varepsilon_{j} \sqrt{\varepsilon_{j}^{2}-m_{\beta}^{2}} \Theta\left(\varepsilon_{j}-m_{\beta}\right) .
\end{aligned}
$$

The KATRIN experiment combines a windowless gaseous molecular tritium source with a spectrometer based on the principle of magnetic adiabatic collimation with electrostatic filtering (MAC-E-filter) $[19,20]$. This apparatus can measure the integral tritium $\beta$-spectrum

$$
R(\langle q U\rangle)=R_{\mathrm{bg}}+A_{\mathrm{sig}} N_{\mathrm{T}} \int_{q U}^{E_{0}} R_{\beta}(E) f(E-\langle q U\rangle) d E
$$

which is the convolution of the differential $\beta$-decay electron spectrum $R_{\beta}(E)$ with the response function $f(E-\langle q U\rangle)$. $N_{\mathrm{T}}$ denotes the effective number of tritium atoms, $R_{\mathrm{bg}}$ is the energy-independent background rate and $A_{\text {sig }}$ is the signal amplitude. The response function defines the probability of passing the MAC-E-filter for an electron with the kinetic energy $E$ at the retarding potential energy $q U .\langle q U\rangle$ is the average over different pixels and scans and serves as the working variable of the integral electron spectrum. The response function used in our analysis is taken from the red curve of the top panel of figure 2 in ref. [1]. Note that an energy resolution of $2.8 \mathrm{eV}$, which is determined by the energy filter width at the minimal and maximal magnetic fields, has been included in the response function. Moreover, an additional Gaussian smearing of $0.25 \mathrm{eV}$ is also included to account for the average effect of $\langle q U\rangle$.

For the analysis of the KATRIN data, we considered the $\chi^{2}$ function

$$
\chi^{2}=\sum_{i=1}^{N}\left(\frac{R_{i}^{\mathrm{obs}}-R_{i}^{\mathrm{pred}}\left(m_{\beta}^{2}+\delta m_{\beta}^{2}\right)}{\sigma_{i}}\right)^{2}+\left(\frac{\delta m_{\beta}^{2}}{0.32}\right)^{2},
$$

where $R_{i}^{\text {obs }}$ and $\sigma_{i}$ are the experimental rate and its statistical uncertainty corresponding to each retarding energy value $\langle q U\rangle_{i}$ in the upper panel of figure 3 in ref. [1]. $R_{i}^{\text {pred }}$ is the predicted rate calculated according to eq. (2.5). The pull term for the variation $\delta m_{\beta}^{2}$ takes into account the systematic uncertainty of $0.32 \mathrm{eV}^{2}$ on $m_{\beta}^{2}$ given in table I of ref. [1]. In the fit we considered four free parameters: $m_{\beta}^{2}$, the endpoint $E_{0}$, the signal amplitude $A_{\text {sig }}$, and the background rate $R_{\mathrm{bg}}$. We calculated the bounds for $m_{\beta}^{2}$ by marginalizing over $E_{0}$, $A_{\text {sig }}$, and $R_{\mathrm{bg}}$. 
In ref. [1], the KATRIN collaboration first analyzed the data allowing negative values of $m_{\beta}^{2}$, as discussed in ref. [21]. With this method, they obtained $m_{\beta}^{2}=-1.0_{-1.1}^{+0.9} \mathrm{eV}^{2}$. Under the same assumption, we obtained $m_{\beta}^{2}=-1.0 \pm 0.9 \mathrm{eV}^{2}$, which is approximately consistent with the official KATRIN result.

In order to calculate the upper bound on the absolute scale of neutrino masses in the framework of three-neutrino mixing, we considered only physical positive values of $m_{\beta}^{2}$, as done by the KATRIN collaboration [1]. We obtained

$$
m_{\beta}<0.8(0.9) \mathrm{eV} \text { at } 90 \%(95 \%) \mathrm{CL},
$$

that nicely coincide with the bounds that the KATRIN collaboration obtained [1] using the Feldman-Cousins method [22].

The approximate agreement of our results for $m_{\beta}$ in the standard framework of threeneutrino mixing with those of the KATRIN collaboration validates our analysis of the KATRIN data.

\section{$3 \quad 3+1$ sterile neutrino mixing}

After the successful test of our method of analysis of the KATRIN data in the case of threeneutrino mixing, we consider the extension to $3+1$ active-sterile neutrino mixing with the differential electron spectrum

$$
R_{\beta}(E)=\left(1-\left|U_{e 4}\right|^{2}\right) R_{\beta}\left(E, m_{\beta}\right)+\left|U_{e 4}\right|^{2} R_{\beta}\left(E, m_{4}\right),
$$

where $U$ is the $4 \times 4$ unitary mixing matrix, $R_{\beta}\left(E, m_{\beta}\right)$ is the three-neutrino differential electron spectrum in eq. (2.4) with $m_{\beta}$ redefined by ${ }^{1}$

$$
m_{\beta}^{2}=\sum_{k=1}^{3} \frac{\left|U_{e k}\right|^{2}}{1-\left|U_{e 4}\right|^{2}} m_{k}^{2},
$$

and $R_{\beta}\left(E, m_{4}\right)$ has the same expression with $m_{\beta}$ replaced by $m_{4}$. We will compare the results of our analysis of the KATRIN data with the results of short-baseline (SBL) reactor antineutrino oscillation experiments, that probe the effective SBL survival probability

$$
\underset{\substack{(-)(-) \\ \nu_{e} \rightarrow \nu_{e}}}{\mathrm{SBL}}=1-\sin ^{2} 2 \vartheta_{e e} \sin ^{2}\left(\frac{\Delta m_{41}^{2} L}{4 E}\right)
$$

where $\Delta m_{i j}^{2}=m_{i}^{2}-m_{j}^{2}, \sin ^{2} 2 \vartheta_{e e}=4\left|U_{e 4}\right|^{2}\left(1-\left|U_{e 4}\right|^{2}\right), L$ is the source-detector distance, and $E$ is the neutrino energy. Note that neutrino oscillation experiments are sensitive to the squared-mass difference ${ }^{2} \Delta m_{41}^{2} \simeq \Delta m_{42}^{2} \simeq \Delta m_{43}^{2}$, whereas the KATRIN experiment is

\footnotetext{
${ }^{1}$ The necessity of the factor $\left(1-\left|U_{e 4}\right|^{2}\right)$ in front of $R_{\beta}\left(E, m_{\beta}\right)$ can be understood by noting that in the limit of negligible masses of $\nu_{1}, \nu_{2}$, and $\nu_{3}$, their contribution to the electron spectrum is given by eq. (2.2) with $m_{1}=m_{2}=m_{3}=0$. In this case one can extract a common mixing factor $\sum_{i=1}^{3}\left|U_{e i}\right|^{2}=1-\left|U_{e 4}\right|^{2}$ and write the contribution of $\nu_{1}, \nu_{2}$, and $\nu_{3}$ as $\left(1-\left|U_{e 4}\right|^{2}\right) R_{\beta}(E, 0)$.

${ }^{2}$ The effective SBL survival probability (3.3) is derived under the approximation $\Delta m_{41}^{2} \simeq \Delta m_{42}^{2} \simeq$ $\Delta m_{43}^{2} \gtrsim 0.1 \mathrm{eV}^{2}$, taking into account the smallness of the values of $\Delta m_{21}^{2}$ and $\left|\Delta m_{31}^{2}\right| \simeq\left|\Delta m_{32}^{2}\right|$, given in eqs. (3.4) and (3.5), respectively.
} 
sensitive to $m_{\beta}$ and $m_{4}$. Therefore, in order to compare the respective results one must make some assumption on the value of one of the three light neutrino masses $\left(m_{1}, m_{2}, m_{3}\right)$, that fixes the value of $m_{\beta}$ through the precise knowledge of the values of the three-neutrino mixing parameters obtained by global fits of solar, atmospheric and long-baseline neutrino oscillation data [23-26]:

$$
\begin{aligned}
\Delta m_{21}^{2} & \simeq 7.5 \times 10^{-5} \mathrm{eV}^{2}, \\
\left|\Delta m_{31}^{2}\right| & \simeq\left|\Delta m_{32}^{2}\right| \simeq 2.5 \times 10^{-3} \mathrm{eV}^{2}, \\
\left|U_{e 2}\right|^{2} & \simeq 0.3 \\
\left|U_{e 3}\right|^{2} & \simeq 0.022,
\end{aligned}
$$

with positive and negative $\Delta m_{31}^{2} \simeq \Delta m_{32}^{2}$ in the two possible cases of Normal Ordering (NO) and Inverted Ordering (IO) of the three light neutrino masses, respectively (see the recent review in ref. [27]). Equation (3.2) can be written as

$$
m_{\beta}^{2}=m_{1}^{2}+\frac{\left|U_{e 2}\right|^{2} \Delta m_{21}^{2}+\left|U_{e 3}\right|^{2} \Delta m_{31}^{2}}{1-\left|U_{e 4}\right|^{2}} .
$$

Hence, we have

$$
\begin{array}{ll}
\text { NO: } \quad m_{\beta}^{2} \simeq m_{1}^{2}+\frac{7.8 \times 10^{-5} \mathrm{eV}^{2}}{1-\left|U_{e 4}\right|^{2}}, \\
\text { IO: } \quad m_{\beta}^{2} \simeq m_{1}^{2}-\frac{3.3 \times 10^{-5} \mathrm{eV}^{2}}{1-\left|U_{e 4}\right|^{2}} .
\end{array}
$$

Therefore, taking into account that the sensitivity of KATRIN to $m_{\beta}^{2}$ is at the level of the $\mathrm{eV}^{2}$ and considering ${ }^{3}\left|U_{e 4}\right|^{2}<0.5$ for $\sin ^{2} 2 \vartheta_{e e}<1$, we can neglect the small deviations of $m_{\beta}^{2}$ from $m_{1}^{2}$ in eqs. (3.9) and (3.10), and consider the approximate relation

$$
\Delta m_{41}^{2} \simeq m_{4}^{2}-m_{\beta}^{2} .
$$

We performed two analyses of the KATRIN data in the framework of $3+1$ active-sterile neutrino mixing. First, we fitted the data considering $A_{\mathrm{sig}}, R_{\mathrm{bg}}, E_{0}, m_{\beta},\left|U_{e 4}\right|^{2}$, and $m_{4}$ as free parameters and we calculated the "free $m_{\beta}$ " confidence level contours in the $\left(\sin ^{2} 2 \vartheta_{e e}, \Delta m_{41}^{2}\right)$ plane shown in figure 1 marginalizing the $\chi^{2}$ over $A_{\mathrm{sig}}, R_{\mathrm{bg}}, E_{0}$ and $m_{\beta}$. This is the most general bound on $3+1$ mixing given by the KATRIN data. We also calculated the confidence level contours in the case of a negligible $m_{\beta}$, shown by the $m_{\beta}=0$ lines in figure 1. This is a reasonable assumption motivated by the likeliness of a neutrino mass hierarchy, with $m_{1,2,3} \ll m_{4}$. It is also useful for the comparison in figure 1 of the KATRIN bounds with the exclusion curves of the Mainz [5] and Troitsk [6, 7] experiments obtained in ref. [8] under the same assumption. One can see from figure 1 that the KATRIN bounds obtained with free $m_{\beta}$ and $m_{\beta}=0$ are slightly different only around $\Delta m_{41}^{2} \approx 200-300 \mathrm{eV}^{2}$, where the Mainz+Troitsk bound is dominant. Therefore, in the following we can safely consider only the analysis of KATRIN data with $m_{\beta}=0$.

\footnotetext{
${ }^{3}$ Since $3+1$ active-sterile neutrino mixing is allowed only as a perturbation of standard three-neutrino mixing, $\left|U_{e 4}\right|^{2}$ cannot be large (see the recent reviews in refs. [2-4]).
} 


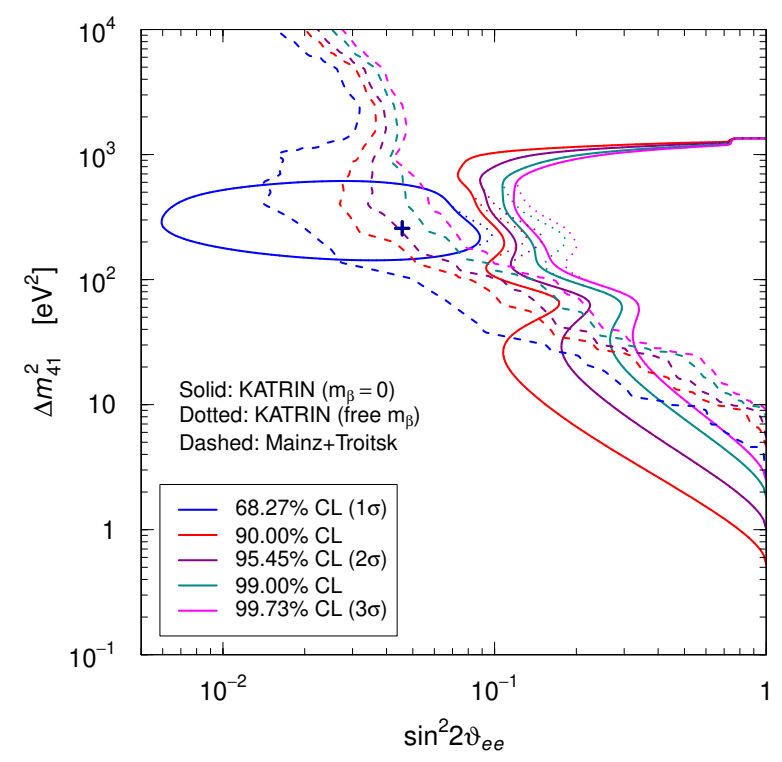

Figure 1. Confidence level contours in the $\left(\sin ^{2} 2 \vartheta_{e e}, \Delta m_{41}^{2}\right)$ plane obtained from the analysis of KATRIN data with $m_{\beta}=0$ (solid) and free $m_{\beta}$ (dotted), and from the results of the Mainz [5] and Troitsk $[6,7]$ experiments [8]. The blue cross indicates the KATRIN best-fit point.

Figure 1 shows that the analysis of the KATRIN data yields a $1 \sigma$ allowed region with non-zero active-sterile neutrino mixing around the best-fit point at $\sin ^{2} 2 \vartheta_{e e}=0.046$ and $\Delta m_{41}^{2}=257 \mathrm{eV}^{2}$. Since the $1 \sigma$ KATRIN allowed region is in tension with the Mainz+Troitsk bound, it is likely due to a statistical fluctuation. Therefore, in the following we will consider only the KATRIN exclusion curves at higher confidence level. However, the slight tension between KATRIN and Mainz+Troitsk has effects on the combined bound that will be discussed below.

Figure 1 shows that the KATRIN data allow us to extend the Mainz+Troitsk excluded region at large mixing to smaller value of $\Delta m_{41}^{2}$, reaching the interesting values of $\Delta m_{41}^{2}$ below $10 \mathrm{eV}^{2}$. In the logarithmic scale of figure 1 , the KATRIN bounds on $\sin ^{2} 2 \vartheta_{e e}$ have an approximately linear decrease when $\Delta m_{41}^{2}$ increases from about $6 \mathrm{eV}^{2}$ to about $30 \mathrm{eV}^{2}$, that corresponds to $m_{4} \approx 5.5 \mathrm{eV}$. For larger values of $\Delta m_{41}^{2}$, the effect of $\nu_{4}$ on the electron spectrum occurs at a distance from the end point for which the data are less constraining. This leads to oscillations of the bounds from $\Delta m_{41}^{2} \approx 30 \mathrm{eV}^{2}$ to $\Delta m_{41}^{2} \approx 10^{3} \mathrm{eV}^{2}$, that corresponds to the value $m_{4} \approx 32 \mathrm{eV}$ for which the data become completely ineffective.

Figure 2 shows the combined $90 \%$ and $99 \%$ CL bounds of the tritium experiments compared with the corresponding KATRIN and Mainz+Troitsk bounds (and the regions allowed by the reactor antineutrino anomaly to be discussed in section 4). One can see that the combined tritium bound extends the Mainz+Troitsk excluded region at large mixing to values of $\Delta m_{41}^{2}$ below $10 \mathrm{eV}^{2}$. However, the combined tritium bound is less stringent than the KATRIN bound in the small- $\Delta m_{41}^{2}$ range where the KATRIN data are dominant (this occurs much more for the 90\% CL bound than for the 99\% CL bound). This strange behavior is due to the location of the minimum of the KATRIN $\chi^{2}$, that is shown in figure 2 


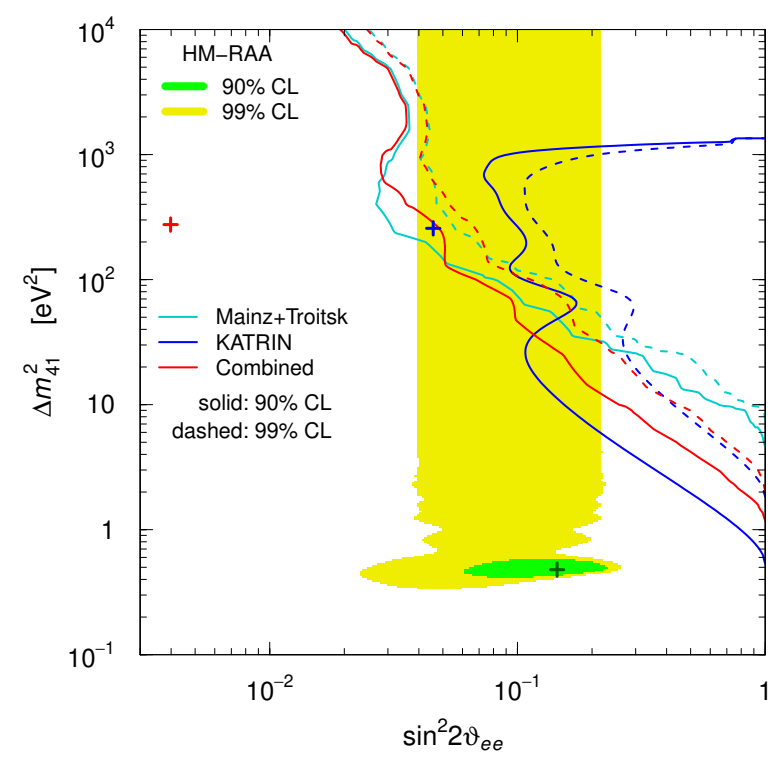

Figure 2. $90 \%$ and $99 \%$ CL exclusion curves in the $\left(\sin ^{2} 2 \vartheta_{e e}, \Delta m_{41}^{2}\right)$ plane obtained from the analysis of KATRIN data with free $m_{\beta}$ and $m_{\beta}=0$. Also shown are the exclusion curves of the Mainz [5] and Troitsk [6, 7] experiments obtained in ref. [8] and the combined exclusion curves. The green and yellow regions are allowed at $90 \%$ and $99 \%$ CL by the neutrino oscillation solution [28] of the Huber-Muller reactor antineutrino anomaly (HM-RAA). The crosses indicate the best-fit points.

by the blue cross, in a point where the Mainz+Troitsk $\chi^{2}$ is not small. The location of the minimum $\chi^{2}$ of the combined fit is shown in figure 2 by the red cross and it is obviously larger than the KATRIN $\chi^{2}$ minimum $\left(\Delta \chi^{2}=2.5\right)$, since it lies out of the KATRIN $1 \sigma$ allowed region shown in figure 1 . Since the confidence level contours are determined by the difference of $\chi^{2}$ (given by table 39.2 of ref. [26] for two degrees of freedom) with respect to the minimum corresponding to the chosen confidence level, the increase of the $\chi^{2}$ minimum leads to a shift towards larger values of $\sin ^{2} 2 \vartheta_{e e}$ of the combined tritium bound with respect to the KATRIN bound in the $\Delta m_{41}^{2}$ range where KATRIN is dominant. For a similar reason, the combined tritium bound is less stringent than the Mainz+Troitsk bound for $\Delta m_{41}^{2} \approx 100-600 \mathrm{eV}^{2}$, where the Mainz+Troitsk bound is dominant.

\section{The reactor antineutrino anomaly}

In figure 2 we have also drawn the regions allowed by the reactor antineutrino anomaly (HM-RAA) [9] according to the recent analysis in ref. [28] of reactor antineutrino data compared with the Huber-Muller prediction [10, 11] (see also ref. [29]). One can see that the combined constraints of tritium-decay experiments can exclude the large- $\Delta m_{41}^{2}$ part of the RAA $99 \%$ allowed region, but it is still too weak to affect the $90 \%$ allowed region around the best-fit point. Note that this HM-RAA region is different from the original reactor antineutrino anomaly allowed region in ref. [9] (see also ref. [30]) mainly because it takes into account only the measured reactor antineutrino rates, without the Bugey-3 [31] $14 \mathrm{~m} / 15 \mathrm{~m}$ spectral ratio that were included in refs. [9, 30]. As nicely illustrated in figure 1 
of ref. [32], the Bugey-3 spectral ratio excludes large mixing for $\Delta m_{41}^{2} \lesssim 2 \mathrm{eV}^{2}$, moving the best-fit region from $\Delta m_{41}^{2} \approx 0.5 \mathrm{eV}^{2}$ to $\Delta m_{41}^{2} \approx 1.8 \mathrm{eV}^{2}$. However, in discussing the reactor antineutrino anomaly it is better to separate the model-dependent anomaly based on the absolute neutrino rate measurements and the model-independent implications of the spectral-ratio measurements.

Recently, also the new reactor antineutrino experiments DANSS [33, 34], PROSPECT [35], and STEREO [36, 37] measured the reactor antineutrino spectrum at different distances. Moreover, the NEOS [38] experiments presented the results of a measurement of the reactor antineutrino spectrum at $24 \mathrm{~m}$ from a reactor, relative to the spectrum measured at about $500 \mathrm{~m}$ by the Daya Bay near detectors [39]. These measurements provide information on short-baseline neutrino oscillations that are independent of the theoretical calculation of the reactor antineutrino flux. Therefore, they can test the model-dependent reactor antineutrino anomaly and their results can be combined with the bounds given by the tritium experiments. Here we consider the published results of the Bugey-3 [31], NEOS [38], and PROSPECT [35] experiments, together with the preliminary 2019 results of the DANSS [34] experiment, that improve significantly the published 2018 results [34]. We cannot include in the analysis the results of the STEREO [36, 37] experiment, because there is not enough available information. For the Bugey-3 experiment we used the same analysis that we used in previous papers [28, 40, 41]. For the NEOS experiment we use the $\chi^{2}$ table kindly provided by the NEOS collaboration. For the PROSPECT experiment we use the $\chi^{2}$ table published as "Supplemental Material" of ref. [35]. For the DANSS experiment we performed an approximate least-square analysis of the 2019 data presented in figure 5 of ref. [34] that reproduces approximately the DANSS exclusion curves in figure 6 of the same paper.

Figure 3 shows the contours of the $2 \sigma$ regions in the $\left(\sin ^{2} 2 \vartheta_{e e}, \Delta m_{41}^{2}\right)$ plane obtained from the reactor spectral ratio measurements of the Bugey-3, NEOS, PROSPECT and DANSS experiments, and the regions allowed at $1 \sigma, 2 \sigma$, and $3 \sigma$ by the combined fit. One can see that there is an indication in favor of short-baseline oscillations at the level of about $2 \sigma$, that is due to the coincidence of the NEOS and DANSS allowed regions at $\Delta m_{41}^{2} \approx 1.3 \mathrm{eV}^{2}$, where there is the best-fit point of the combined fit for $\sin ^{2} 2 \vartheta_{e e}=0.026$ and $\Delta m_{41}^{2}=1.3 \mathrm{eV}^{2}$. The NEOS and DANSS allowed regions partially overlap also at $\Delta m_{41}^{2} \approx 0.4 \mathrm{eV}^{2}$, where there is a combined $1 \sigma$-allowed region, and at $\Delta m_{41}^{2} \approx 3 \mathrm{eV}^{2}$, where there is a tiny combined $2 \sigma$-allowed region. This model-independent indication in favor of short-baseline oscillations was discussed in refs. [41, 42] using the 2018 [33] DANSS data and in ref. [29] using both the 2018 and the 2019 [34] DANSS data. Here, as explained above, we use the 2019 DANSS data, that lead to a diminished indication in favor of shortbaseline oscillations with respect to the 2018 DANSS data. Indeed, from the combined NEOS and DANSS analyses we find only a $2.6 \sigma$ indication of short-baseline oscillations, that is smaller than the $3.7 \sigma$ obtained in ref. [41]. These values agree approximately with those found in ref. [29].

Figure 4 shows the $99 \%$ exclusion curve in the $\left(\sin ^{2} 2 \vartheta_{e e}, \Delta m_{41}^{2}\right)$ plane obtained from the combined analysis of the Bugey-3, NEOS, PROSPECT and DANSS spectral ratios, that constrain the mixing for low values of $\Delta m_{41}^{2}$, together with the combined $99 \% \mathrm{CL}$ 


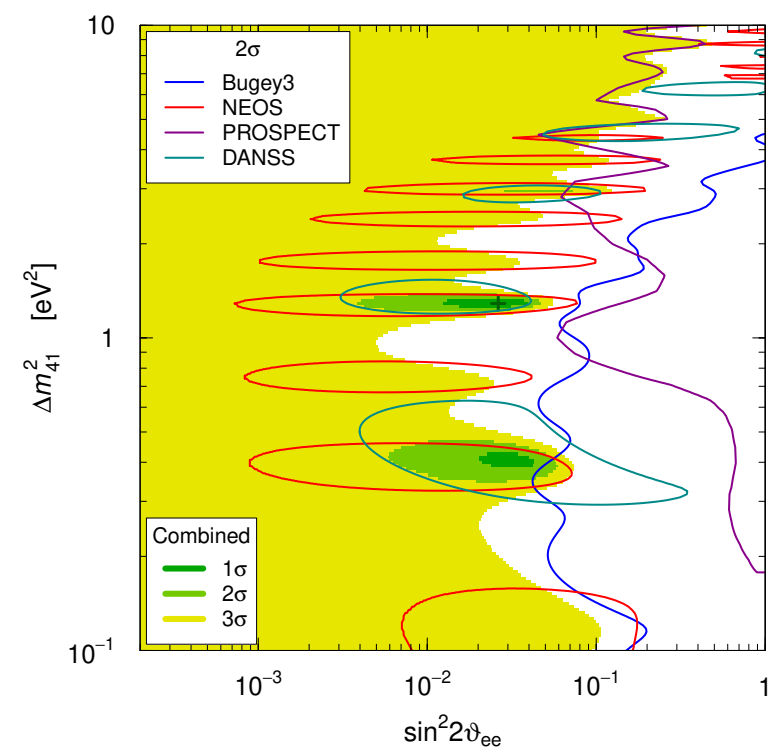

Figure 3. Contours of the $2 \sigma$ regions in the $\left(\sin ^{2} 2 \vartheta_{e e}, \Delta m_{41}^{2}\right)$ plane obtained from the reactor spectral ratio measurements of the Bugey-3, NEOS, PROSPECT and DANSS experiments. The shadowed regions are allowed by the combined fit, with the best fit point indicated by the cross.

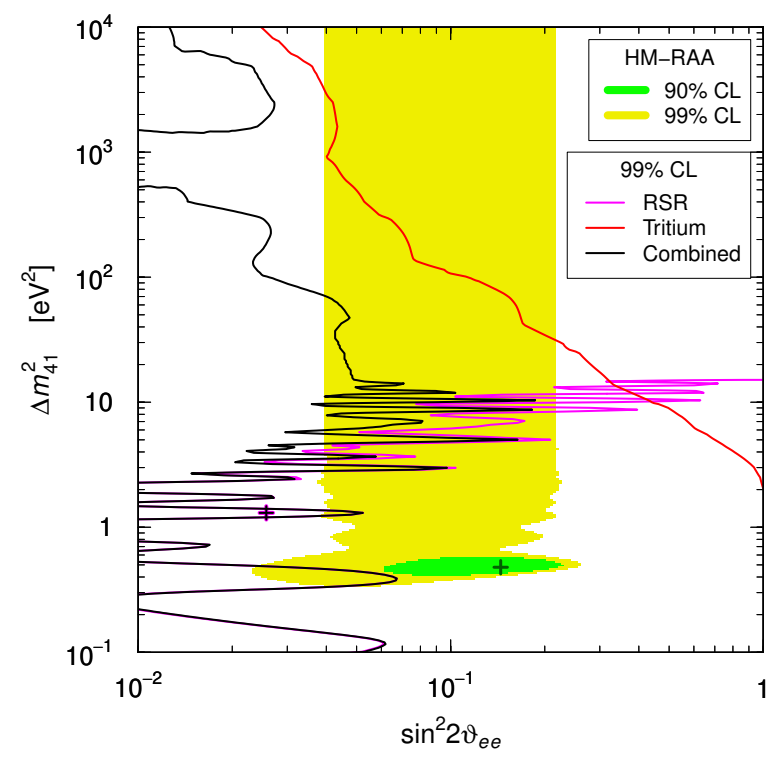

Figure 4. $99 \%$ CL exclusion curves in the $\left(\sin ^{2} 2 \vartheta_{e e}, \Delta m_{41}^{2}\right)$ plane obtained from the analysis of the data of the Mainz, Troitsk and KATRIN tritium experiments and the combined analysis of the reactor spectral ratio (RSR) measurements of the Bugey-3, NEOS, PROSPECT and DANSS experiments. Also shown is the combined tritium and reactor spectral-ratio exclusion curve and the regions allowed at 90\% and 99\% CL by the neutrino oscillation solution [28] of the Huber-Muller reactor antineutrino anomaly (HM-RAA). The crosses indicate the best-fit points. 
exclusion curve of the Mainz, Troitsk and KATRIN tritium experiments, that constrains the mixing for large values of $\Delta m_{41}^{2}$. Figure 4 shows also the combined tritium and reactor spectral-ratio 99\% CL exclusion curve, that disfavors most of the 99\% CL allowed region [28] of the Huber-Muller reactor antineutrino anomaly. Note that the combined tritium and reactor spectral-ratio bound at large values of $\Delta m_{41}^{2}$ is much more stringent than the tritium bound, in spite of the lack of sensitivity of the reactor spectral ratio experiments for $\Delta m_{41}^{2} \gtrsim 10 \mathrm{eV}^{2}$. The reason is that the $99 \%$ exclusion curve is determined by the appropriate difference of $\chi^{2}$ (given by table 39.2 of ref. [26] for two degrees of freedom) with respect to the global $\chi^{2}$ minimum that occurs at $\sin ^{2} 2 \vartheta_{e e}=0.026$ and $\Delta m_{41}^{2}=1.3 \mathrm{eV}^{2}$. This point practically coincides with the reactor spectral ratio best fit in figure 3 and is far away from the tritium-only best fit in figure 2, because the reactor spectral ratio data are dominant in the combined fit. The stringent combined tritium and reactor spectralratio bound at large values of $\Delta m_{41}^{2}$ is due to the combined effects of the tritium bound in that region and the reactor spectral ratio data that allow these large values of $\Delta m_{41}^{2}$ only at $3 \sigma$, as can be seen in figure 3 . The reactor spectral ratio data $\chi^{2}$ has this effect for large values of $\Delta m_{41}^{2}$, where the experiments are not sensitive, because the data prefer the small- $\Delta m_{41}^{2}$ region near the best fit. This is an obviously correct effect if one thinks that the measurement of a physical quantity in an experiment excludes all the values of the physical quantity that are enormously different from the measured one and for which the experiment is not sensitive.

Figure 4 shows that there is a tension between the active-sterile oscillations indicated by the Huber-Muller reactor antineutrino anomaly and the combined bound obtained from tritium and reactor spectral-ratio measurements. However, it is likely that the HuberMuller antineutrino flux prediction must be revised, as indicated by the observation of a large spectral distortion at $5 \mathrm{MeV}$ in the RENO [43, 44], Double Chooz [45], Daya Bay [39], and NEOS [38] experiments (see the reviews in refs. [46, 47]). As already discussed in ref. [29], there are two recent reactor antineutrino flux calculations that may improve the Huber-Muller prediction: the calculation of Estienne, Fallot et al. (EF) [12] that is based on the summation method, and the calculation of Hayen, Kostensalo, Severijns, Suhonen (HKSS) [13] that improves the conversion method by including the effects of forbidden $\beta$ decays through shell-model calculations. Unfortunately, as discussed in ref. [29], a comparison of the results of the two new calculations does not lead to a clarification of the problem of the reactor antineutrino anomaly, because the corresponding antineutrino flux predictions diverge: the EF calculation resulted in a ${ }^{235} \mathrm{U}$ antineutrino flux prediction that is smaller than the HM prediction, leading to a decrease of the reactor antineutrino anomaly, whereas the HKSS fluxes are larger than the HM fluxes, leading to an increase of the reactor antineutrino anomaly. Figure 5 show a comparison of the bounds in the $\left(\sin ^{2} 2 \vartheta_{e e}, \Delta m_{41}^{2}\right)$ plane obtained from the tritium experiments and the reactor spectral ratios with the regions allowed by the fits of the absolute reactor rates assuming the EF and HKSS fluxes. We took into account the uncertainties of the HKSS fluxes given in ref. [13]. On the other hand, since the EF cross sections per fission are given in ref. [12] without the associated uncertainties, for them we adopted the uncertainties associated with the summation spectra estimated in ref. [48]: $5 \%$ for ${ }^{235} \mathrm{U},{ }^{239} \mathrm{Pu}$, and ${ }^{241} \mathrm{Pu}$, and $10 \%$ for ${ }^{238} \mathrm{U}$. 


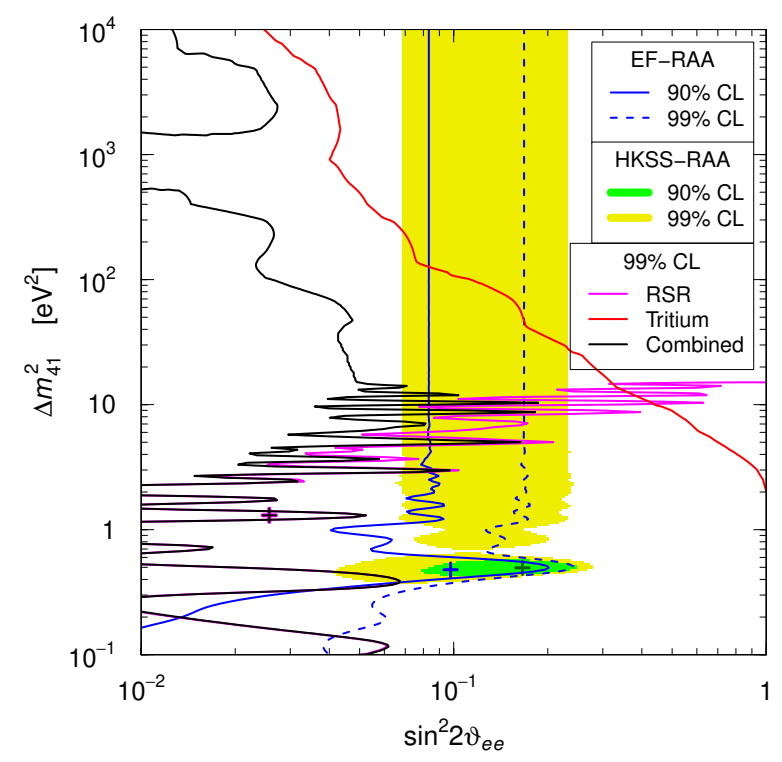

Figure 5. 99\% CL exclusion curves in the $\left(\sin ^{2} 2 \vartheta_{e e}, \Delta m_{41}^{2}\right)$ plane obtained from the combined analysis of the data of the Mainz, Troitsk and KATRIN tritium experiments and the combined analysis of the reactor spectral ratio (RSR) measurements of the Bugey-3, NEOS, PROSPECT and DANSS experiments. Also shown is the combined tritium and reactor spectral-ratio exclusion curve and the regions allowed at $90 \%$ and $99 \%$ CL by the fits of the absolute reactor rates assuming the Estienne, Fallot et al. [12] (EF-RAA) and the Hayen, Kostensalo, Severijns, Suhonen [13] (HKSSRAA) reactor antineutrino fluxes. The crosses indicate the best-fit points.

From figure 5, one can see that the EF antineutrino flux calculation leads only to an upper bound on the mixing at $90 \% \mathrm{CL}$ and higher. Therefore, in this case the reactor antineutrino anomaly is not statistically significant and the EF-RAA upper bound is compatible with the upper bounds obtained from the tritium experiments and the reactor spectral ratios.

On the other hand, the HKSS fluxes lead to an increase of the reactor antineutrino anomaly with respect to the HM prediction and the corresponding HKSS-RAA allowed regions in figure 5 are limited to larger mixing than the HM-RAA allowed regions in figure 4. Therefore, the tension of the HKSS-RAA with the tritium and reactor spectral ratios bounds is larger than that of the HM-RAA. From figure 5 one can see that only very small portions of the HKSS-RAA 99\% allowed region are not excluded by the combined $99 \%$ bound of the tritium experiments and the reactor spectral ratios.

\section{Neutrino-4}

Let us now consider the results of the Neutrino-4 reactor experiment [14], that is another experiment that measured the ratios of the spectra at different distances from the reactor, between 6 and $12 \mathrm{~m}$. We did not consider it so far because the result of this experiment is an anomalous indication of short-baseline oscillations with large mixing that is in tension with all the other experimental results. This can be seen in figure 6 , where we compare 


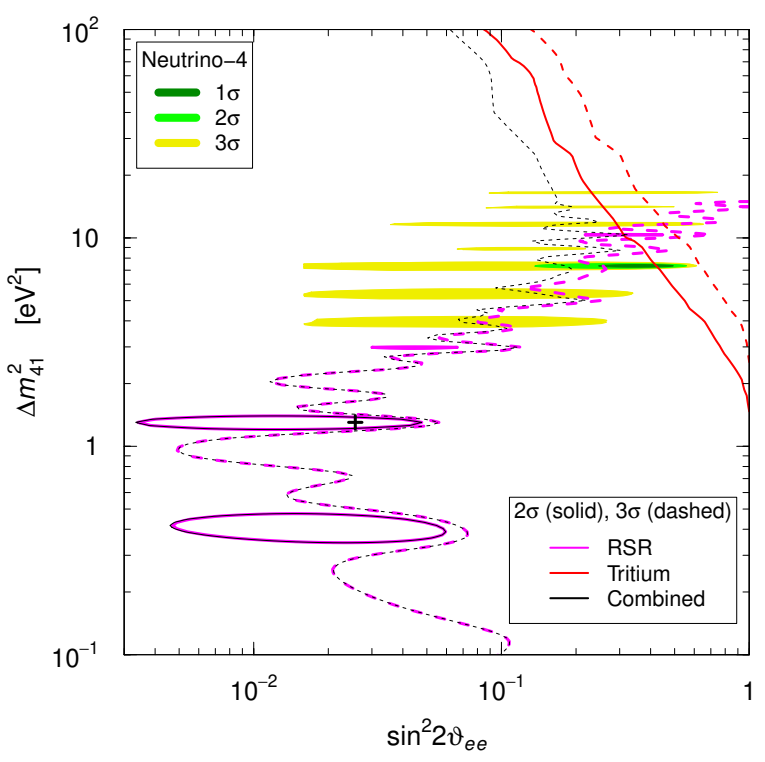

Figure 6. $2 \sigma$ and $3 \sigma$ bounds in the $\left(\sin ^{2} 2 \vartheta_{e e}, \Delta m_{41}^{2}\right)$ plane obtained from the combined analysis of the data of the Mainz, Troitsk and KATRIN tritium experiments and the combined analysis of the reactor spectral ratio (RSR) measurements of the Bugey-3, NEOS, PROSPECT and DANSS experiments. Also shown are the combined tritium and reactor spectral-ratio bounds (with the best fit indicated by the black cross) and the regions allowed at $1 \sigma, 2 \sigma$, and $3 \sigma$ by the results of the Neutrino-4 reactor experiment [14].

the bounds in the $\left(\sin ^{2} 2 \vartheta_{e e}, \Delta m_{41}^{2}\right)$ plane obtained from the tritium experiments and the reactor spectral ratios with the allowed regions of the Neutrino-4 reactor experiment [14].

At $2 \sigma$, the combination of the reactor spectral-ratio and tritium measurements have allowed regions at $\Delta m_{41}^{2} \approx 1.3 \mathrm{eV}^{2}$, where there is the best-fit point for $\sin ^{2} 2 \vartheta_{e e}=0.026$ and $\Delta m_{41}^{2}=1.3 \mathrm{eV}^{2}$, and at $\Delta m_{41}^{2} \approx 0.4 \mathrm{eV}^{2}$, that correspond to those in figure 3 and are due to the coincidence of the NEOS and DANSS allowed regions discussed above. Therefore, all the $3 \sigma$ Neutrino- 4 allowed regions are excluded at $2 \sigma$ by the reactor spectral-ratio and tritium measurements.

Moreover, the large- $\sin ^{2} 2 \vartheta_{e e}$ parts of the $3 \sigma$ Neutrino- 4 allowed regions and most of the $2 \sigma$ Neutrino- 4 allowed region are excluded by the combined $3 \sigma$ tritium and reactor spectral ratio bound.

\section{The gallium neutrino anomaly}

Let us finally consider the gallium neutrino anomaly [30, 52-58], that is a short-baseline disappearance of $\nu_{e}$ 's found in the gallium radioactive source experiments GALLEX [59-61] and SAGE [53, 62-64]. There is some uncertainty on the magnitude of the gallium neutrino anomaly, that depends on the detection cross section, which must be calculated, as in refs. [50, 52], or extrapolated from measurements of $(p, n)[49,65]$ or $\left({ }^{3} \mathrm{He},{ }^{3} \mathrm{H}\right)[51]$ chargeexchange reactions. Figure 7 shows the regions in the $\left(\sin ^{2} 2 \vartheta_{e e}, \Delta m_{41}^{2}\right)$ plane allowed at $90 \%$ CL by the gallium neutrino anomaly using the detection cross sections considered 


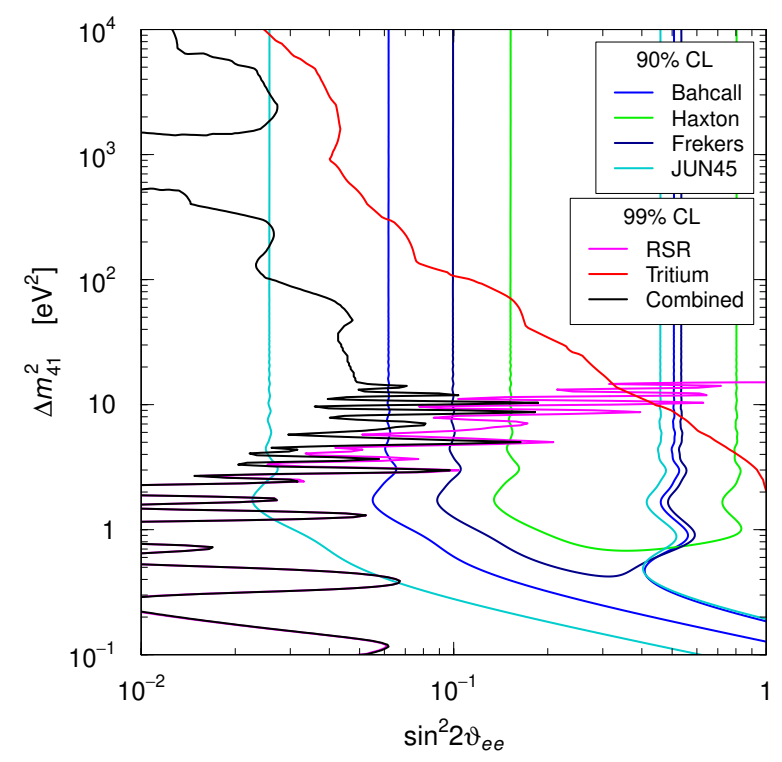

Figure 7. Comparison of the regions in the $\left(\sin ^{2} 2 \vartheta_{e e}, \Delta m_{41}^{2}\right)$ plane allowed at $90 \%$ CL by the gallium neutrino anomaly using the Bahcall [49], Haxton [50], Frekers [51], and JUN45 [52] neutrino detection cross sections discussed in ref. [52] with the 99\% CL exclusion curves obtained from the combined analysis of the data of the Mainz, Troitsk and KATRIN tritium experiments and the combined analysis of the reactor spectral ratio (RSR) measurements of the Bugey-3, NEOS, PROSPECT and DANSS experiments. Also shown is the combined tritium and reactor exclusion curve.

recently in ref. [52], where a new shell model calculation based on the effective Hamiltonian JUN45 was presented. The Bahcall cross section was derived in ref. [49] from the $(p, n)$ charge-exchange measurements in ref. [65]. The Haxton cross section was calculated in ref. [50] using a shell model. The Frekers cross section was obtained from the $\left({ }^{3} \mathrm{He},{ }^{3} \mathrm{H}\right)[51]$ charge-exchange measurements in ref. [51].

As done in ref. [52], we show in figure 7 the contours of the 90\% CL allowed regions that have a lower bound for the effective mixing parameter $\sin ^{2} 2 \vartheta_{e e}$. One can see that the relatively large Haxton cross section gives the strongest anomaly, which requires rather large active sterile mixing and is in severe tension with the tritium and reactor spectral ratio bounds. Almost all the 90\% CL Haxton allowed region is excluded at 99\% CL by the combined tritium and reactor spectral ratio bound. The smaller Frekers and Bahcall cross sections allow smaller values of the mixing, but the corresponding 90\% CL allowed regions in figure 7 are in tension with the combined tritium and reactor spectral ratio 99\% CL exclusion curve, with only some very small not-excluded areas. The JUN45 cross section is the smallest one and allows the smallest mixing, as one can see from figure 7 , where the corresponding 90\% CL allowed region has several areas that are not excluded by the combined $99 \%$ CL tritium and reactor spectral ratio bound. In particular, there is a large not-excluded area at large values of $\Delta m_{41}^{2}$, between about 5 and $100 \mathrm{eV}^{2}$. These comparisons indicate that the smallest JUN45 gallium detection cross section is favored with respect to the others. Note that the KATRIN bound is essential for the exclusion of 
large parts of the gallium allowed regions for $\Delta m_{41}^{2}$ between about 10 and $80 \mathrm{eV}^{2}$, where the KATRIN data dominate the tritium bound.

\section{Conclusions}

In this paper we have discussed the implications for $3+1$ active-sterile neutrino mixing of the recent KATRIN data [1] on the search for the absolute value of neutrino masses. We have first analyzed the KATRIN data in the framework of standard three-neutrino mixing, in order to check the validity of our method by comparing the resulting bound on the effective mass $m_{\beta}$ with that obtained by the KATRIN collaboration. Then, we have presented the bounds obtained from the analysis of the KATRIN data on the shortbaseline oscillation parameters $\sin ^{2} 2 \vartheta_{e e}$ and $\Delta m_{41}^{2}$ in the framework of $3+1$ active-sterile neutrino mixing. We have shown that the KATRIN data allow to improve the bounds of the Mainz [5] and Troitsk [6, 7] experiments discussed in ref. [8] extending the excluded region from $\Delta m_{41}^{2} \approx 10-100 \mathrm{eV}^{2}$ to $\Delta m_{41}^{2} \approx 1-10 \mathrm{eV}^{2}$ for large mixing $\left(\sin ^{2} 2 \vartheta_{e e} \gtrsim 0.1\right)$. This result allows us to extend the exclusion of the large- $\Delta m_{41}^{2}$ solution of the HuberMuller reactor antineutrino anomaly to $\Delta m_{41}^{2} \approx 10 \mathrm{eV}^{2}$ for $\sin ^{2} 2 \vartheta_{e e} \approx 0.1$ at $90 \% \mathrm{CL}$ (see figure 2).

We also considered the model-independent bounds of the Bugey-3 [31], NEOS [38], PROSPECT [35], and DANSS [33, 34] experiments that measured the reactor antineutrino spectrum at different distances. We have shown that there is a persistent modelindependent indication [29, 41, 42] of short-baseline oscillations due to the coincidence of the NEOS and DANSS allowed regions, albeit with a smaller statistical significance passing from the 2018 [33] to the 2019 [34] DANSS data, in agreement with the discussion in ref. [29].

The combination of the bounds of the reactor spectral ratio measurements excludes most of the low- $\Delta m_{41}^{2}$ solution of the Huber-Muller reactor antineutrino anomaly. Therefore, combining the tritium and reactor spectral ratio bounds, we are able to exclude most of the region in the $\left(\sin ^{2} 2 \vartheta_{e e}, \Delta m_{41}^{2}\right)$ plane corresponding to the short-baseline solution of the Huber-Muller reactor antineutrino anomaly (see figure 4).

We also discussed the implications of these bounds for the interpretations of the absolute reactor antineutrino rates assuming one of the two recent new reactor antineutrino flux calculations by Estienne, Fallot et al. (EF) [12] and Hayen, Kostensalo, Severijns, Suhonen (HKSS) [13]. We have shown that the EF calculation, that predicts a ${ }^{235} \mathrm{U}$ antineutrino flux that is smaller than that of Huber-Muller, is in agreement with the bounds on $3+1$ mixing obtained from the tritium and reactor spectral ratio measurements. On the other hand, since the HKSS calculation predicts reactor antineutrino fluxes that are larger than those of Huber-Muller, the HKSS antineutrino anomaly region in the $\left(\sin ^{2} 2 \vartheta_{e e}, \Delta m_{41}^{2}\right)$ plane is more excluded than the Huber-Muller one (see figure 5).

We also compared the tritium and reactor spectral ratio bounds on $3+1$ mixing with the indication of large mixing of the Neutrino-4 reactor experiment [14]. We have shown that the Neutrino- 4 allowed regions in the $\left(\sin ^{2} 2 \vartheta_{e e}, \Delta m_{41}^{2}\right)$ plane are excluded at $2 \sigma$ by the other reactor spectral ratio measurements. The $3 \sigma$ combined tritium and reactor 
spectral ratio bound excludes the large- $\sin ^{2} 2 \vartheta_{e e}$ parts of the $3 \sigma$ Neutrino- 4 allowed regions (see figure 6).

We finally considered the gallium neutrino anomaly and we have shown that the combined bound of tritium and reactor spectral ratio measurements favor the recent JUN45 shell model calculation of the neutrino-gallium cross section [52] with respect to older estimates [49-51].

\section{Acknowledgments}

We would like to thank Bryce Littlejohn, Loredana Gastaldo and Francesco Vissani for useful discussions that helped to improve the paper. The work of C. Giunti was partially supported by the research grant "The Dark Universe: A Synergic Multimessenger Approach" number 2017X7X85K under the program PRIN 2017 funded by the Ministero dell'Istruzione, Università e della Ricerca (MIUR). The work of Y.F. Li and Y.Y. Zhang was supported in part by Beijing Natural Science Foundation under Grant No. 1192019, and by the National Natural Science Foundation of China under Grant No. 11835013. Y.F. Li is also grateful for the support by the CAS Center for Excellence in Particle Physics (CCEPP).

Open Access. This article is distributed under the terms of the Creative Commons Attribution License (CC-BY 4.0), which permits any use, distribution and reproduction in any medium, provided the original author(s) and source are credited.

\section{References}

[1] KATRIN collaboration, Improved Upper Limit on the Neutrino Mass from a Direct Kinematic Method by KATRIN, Phys. Rev. Lett. 123 (2019) 221802 [arXiv:1909.06048] [INSPIRE].

[2] C. Giunti and T. Lasserre, eV-scale Sterile Neutrinos, Ann. Rev. Nucl. Part. Sci. 69 (2019) 163 [arXiv: 1901.08330] [INSPIRE].

[3] A. Diaz, C.A. Argüelles, G.H. Collin, J.M. Conrad and M.H. Shaevitz, Where Are We With Light Sterile Neutrinos?, arXiv:1906.00045 [INSPIRE].

[4] S. Böser et al., Status of Light Sterile Neutrino Searches, Prog. Part. Nucl. Phys. 111 (2020) 103736 [arXiv: 1906.01739] [INSPIRE].

[5] C. Kraus, A. Singer, K. Valerius and C. Weinheimer, Limit on sterile neutrino contribution from the Mainz Neutrino Mass Experiment, Eur. Phys. J. C 73 (2013) 2323 [arXiv: 1210.4194] [INSPIRE].

[6] A.I. Belesev et al., An upper limit on additional neutrino mass eigenstate in 2 to $100 \mathrm{eV}$ region from 'Troitsk nu-mass' data, JETP Lett. 97 (2013) 67 [arXiv:1211.7193] [INSPIRE].

[7] A.I. Belesev et al., The search for an additional neutrino mass eigenstate in the 2-100 eV region from 'Troitsk nu-mass' data: a detailed analysis, J. Phys. G 41 (2014) 015001 [arXiv: 1307.5687] [INSPIRE].

[8] C. Giunti, M. Laveder, Y.F. Li and H.W. Long, Short-baseline electron neutrino oscillation length after troitsk, Phys. Rev. D 87 (2013) 013004 [arXiv:1212.3805] [INSPIRE]. 
[9] G. Mention et al., The Reactor Antineutrino Anomaly, Phys. Rev. D 83 (2011) 073006 [arXiv:1101.2755] [INSPIRE].

[10] T.A. Mueller et al., Improved Predictions of Reactor Antineutrino Spectra, Phys. Rev. C 83 (2011) 054615 [arXiv: 1101.2663] [INSPIRE].

[11] P. Huber, On the determination of anti-neutrino spectra from nuclear reactors, Phys. Rev. C 84 (2011) 024617 [Erratum ibid. C 85 (2012) 029901] [arXiv:1106.0687] [INSPIRE].

[12] M. Estienne et al., Updated Summation Model: An Improved Agreement with the Daya Bay Antineutrino Fluxes, Phys. Rev. Lett. 123 (2019) 022502 [arXiv:1904.09358] [INSPIRE].

[13] L. Hayen, J. Kostensalo, N. Severijns and J. Suhonen, First-forbidden transitions in the reactor anomaly, arXiv:1908.08302 [INSPIRE].

[14] NEUTRINO-4 collaboration, First Observation of the Oscillation Effect in the Neutrino-4 Experiment on the Search for the Sterile Neutrino, Pisma Zh. Eksp. Teor. Fiz. 109 (2019) 209 [arXiv: 1809.10561] [INSPIRE].

[15] E.J. Konopinski and G.E. Uhlenbeck, On the Fermi Theory of beta-Radioactivity, Phys. Rev. 48 (1935) 7 [INSPIRE].

[16] P.O. Ludl and W. Rodejohann, Direct Neutrino Mass Experiments and Exotic Charged Current Interactions, JHEP 06 (2016) 040 [arXiv:1603.08690] [INSPIRE].

[17] A. Saenz, S. Jonsell and P. Froelich, Improved Molecular Final-State Distribution of He $\mathrm{T}^{+}$ for the $\beta$-Decay Process of T2, Phys. Rev. Lett. 84 (2000) 242 [INSPIRE].

[18] N. Doss, J. Tennyson, A. Saenz and S. Jonsell, Molecular effects in investigations of tritium molecule beta decay endpoint experiments, Phys. Rev. C 73 (2006) 025502 [INSPIRE].

[19] V.M. Lobashev and P.E. Spivak, A method for measuring the anti-electron-neutrino rest mass, Nucl. Instrum. Meth. A 240 (1985) 305 [INSPIRE].

[20] A. Picard et al., A solenoid retarding spectrometer with high resolution and transmission for keV electrons, Nucl. Instrum. Meth. B 63 (1992) 345.

[21] M. Kleesiek et al., $\beta$-Decay Spectrum, Response Function and Statistical Model for Neutrino Mass Measurements with the KATRIN Experiment, Eur. Phys. J. C 79 (2019) 204 [arXiv: 1806.00369] [INSPIRE].

[22] G.J. Feldman and R.D. Cousins, A unified approach to the classical statistical analysis of small signals, Phys. Rev. D 57 (1998) 3873 [physics/9711021] [INSPIRE].

[23] P.F. de Salas, D.V. Forero, C.A. Ternes, M. Tórtola and J.W.F. Valle, Status of neutrino oscillations 2018: $3 \sigma$ hint for normal mass ordering and improved CP sensitivity, Phys. Lett. B 782 (2018) 633 [arXiv:1708.01186] [INSPIRE].

[24] F. Capozzi, E. Lisi, A. Marrone and A. Palazzo, Current unknowns in the three neutrino framework, Prog. Part. Nucl. Phys. 102 (2018) 48 [arXiv: 1804.09678] [INSPIRE].

[25] I. Esteban, M.C. Gonzalez-Garcia, A. Hernandez-Cabezudo, M. Maltoni and T. Schwetz, Global analysis of three-flavour neutrino oscillations: synergies and tensions in the determination of $\theta_{23}, \delta_{C P}$ and the mass ordering, JHEP 01 (2019) 106 [arXiv:1811.05487] [INSPIRE].

[26] Particle Data Group collaboration, Review of Particle Physics, Phys. Rev. D 98 (2018) 030001 [INSPIRE]. 
[27] P.F. De Salas, S. Gariazzo, O. Mena, C.A. Ternes and M. Tórtola, Neutrino Mass Ordering from Oscillations and Beyond: 2018 Status and Future Prospects, Front. Astron. Space Sci. $\mathbf{5}$ (2018) 36 [arXiv:1806.11051] [INSPIRE].

[28] C. Giunti, Y.F. Li, B.R. Littlejohn and P.T. Surukuchi, Diagnosing the Reactor Antineutrino Anomaly with Global Antineutrino Flux Data, Phys. Rev. D 99 (2019) 073005 [arXiv: 1901.01807] [INSPIRE].

[29] J.M. Berryman and P. Huber, Reevaluating Reactor Antineutrino Anomalies with Updated Flux Predictions, Phys. Rev. D 101 (2020) 015008 [arXiv: 1909.09267] [InSPIRE].

[30] C. Giunti, M. Laveder, Y.F. Li, Q.Y. Liu and H.W. Long, Update of Short-Baseline Electron Neutrino and Antineutrino Disappearance, Phys. Rev. D 86 (2012) 113014 [arXiv: 1210.5715] [INSPIRE].

[31] Y. Declais et al., Search for neutrino oscillations at 15-meters, 40-meters and 95-meters from a nuclear power reactor at Bugey, Nucl. Phys. B 434 (1995) 503 [INSPIRE].

[32] J. Kopp, P.A.N. Machado, M. Maltoni and T. Schwetz, Sterile Neutrino Oscillations: The Global Picture, JHEP 05 (2013) 050 [arXiv: 1303.3011] [INSPIRE].

[33] DANSS collaboration, Search for sterile neutrinos at the DANSS experiment, Phys. Lett. B 787 (2018) 56 [arXiv: 1804.04046] [INSPIRE].

[34] DANSS collaboration, Recent results of the DANSS experiment, in 2019 European Physical Society Conference on High Energy Physics (EPS-HEP2019) Ghent, Belgium, July 10-17, 2019, arXiv:1911.10140 [INSPIRE].

[35] PROSPECT collaboration, First search for short-baseline neutrino oscillations at HFIR with PROSPECT, Phys. Rev. Lett. 121 (2018) 251802 [arXiv: 1806. 02784] [INSPIRE].

[36] STEREO collaboration, Sterile Neutrino Constraints from the STEREO Experiment with 66 Days of Reactor-On Data, Phys. Rev. Lett. 121 (2018) 161801 [arXiv:1806.02096] [INSPIRE].

[37] STEREO collaboration, Improved Sterile Neutrino Constraints from the STEREO Experiment with 179 Days of Reactor-On Data, arXiv:1912.06582 [INSPIRE].

[38] NEOS collaboration, Sterile Neutrino Search at the NEOS Experiment, Phys. Rev. Lett. 118 (2017) 121802 [arXiv:1610.05134] [INSPIRE].

[39] Daya Bay collaboration, Improved Measurement of the Reactor Antineutrino Flux and Spectrum at Daya Bay, Chin. Phys. C 41 (2017) 013002 [arXiv:1607.05378] [INSPIRE].

[40] S. Gariazzo, C. Giunti, M. Laveder and Y.F. Li, Updated Global 3+1 Analysis of Short-BaseLine Neutrino Oscillations, JHEP 06 (2017) 135 [arXiv:1703.00860] [INSPIRE].

[41] S. Gariazzo, C. Giunti, M. Laveder and Y.F. Li, Model-independent $\bar{\nu}_{e}$ short-baseline oscillations from reactor spectral ratios, Phys. Lett. B 782 (2018) 13 [arXiv:1801.06467] [INSPIRE].

[42] M. Dentler et al., Updated Global Analysis of Neutrino Oscillations in the Presence of eV-Scale Sterile Neutrinos, JHEP 08 (2018) 010 [arXiv: 1803.10661] [INSPIRE].

[43] RENO collaboration, New Results from RENO and The 5 MeV Excess, AIP Conf. Proc. 1666 (2015) 080002 [arXiv:1410.7987] [INSPIRE]. 
[44] RENO collaboration, Observation of Energy and Baseline Dependent Reactor Antineutrino Disappearance in the RENO Experiment, Phys. Rev. Lett. 116 (2016) 211801 [arXiv: 1511.05849] [INSPIRE].

[45] Double CHOOZ collaboration, Improved measurements of the neutrino mixing angle $\theta_{13}$ with the Double CHOOZ detector, JHEP 10 (2014) 086 [Erratum ibid. 02 (2015) 074] [arXiv: 1406.7763] [INSPIRE].

[46] P. Huber, Reactor antineutrino fluxes - Status and challenges, Nucl. Phys. B 908 (2016) 268 [arXiv: 1602.01499] [INSPIRE].

[47] A.C. Hayes and P. Vogel, Reactor Neutrino Spectra, Ann. Rev. Nucl. Part. Sci. 66 (2016) 219 [arXiv: 1605. 02047] [INSPIRE].

[48] A.C. Hayes, G. Jungman, E.A. McCutchan, A.A. Sonzogni, G.T. Garvey and X. Wang, Analysis of the Daya Bay Reactor Antineutrino Flux Changes with Fuel Burnup, Phys. Rev. Lett. 120 (2018) 022503 [arXiv: 1707.07728] [INSPIRE].

[49] J.N. Bahcall, Gallium solar neutrino experiments: Absorption cross-sections, neutrino spectra and predicted event rates, Phys. Rev. C 56 (1997) 3391 [hep-ph/9710491] [INSPIRE].

[50] W.C. Haxton, Cross-section uncertainties in the gallium neutrino source experiments, Phys. Lett. B 431 (1998) 110 [nucl-th/9804011] [INSPIRE].

[51] D. Frekers et al., The ${ }^{71} \mathrm{Ga}\left({ }^{3} \mathrm{He}, t\right)$ reaction and the low-energy neutrino response, Phys. Lett. B 706 (2011) 134 [INSPIRE].

[52] J. Kostensalo, J. Suhonen, C. Giunti and P.C. Srivastava, The gallium anomaly revisited, Phys. Lett. B 795 (2019) 542 [arXiv: 1906.10980] [INSPIRE].

[53] J.N. Abdurashitov et al., Measurement of the response of a Ga solar neutrino experiment to neutrinos from an ${ }^{37}$ Ar source, Phys. Rev. C 73 (2006) 045805 [nucl-ex/0512041] [INSPIRE].

[54] M. Laveder, Unbound neutrino roadmaps, Nucl. Phys. Proc. Suppl. 168 (2007) 344 [INSPIRE].

[55] C. Giunti and M. Laveder, Short-Baseline Active-Sterile Neutrino Oscillations?, Mod. Phys. Lett. A 22 (2007) 2499 [hep-ph/0610352] [INSPIRE].

[56] M.A. Acero, C. Giunti and M. Laveder, Limits on $\nu_{e}$ and $\bar{\nu}_{e}$ disappearance from Gallium and reactor experiments, Phys. Rev. D 78 (2008) 073009 [arXiv:0711.4222] [INSPIRE].

[57] C. Giunti and M. Laveder, VSBL Electron Neutrino Disappearance, Phys. Rev. D 80 (2009) 013005 [arXiv: 0902 .1992] [INSPIRE].

[58] C. Giunti and M. Laveder, Statistical Significance of the Gallium Anomaly, Phys. Rev. C 83 (2011) 065504 [arXiv: 1006.3244] [INSPIRE].

[59] GALLEX collaboration, First results from the ${ }^{51} \mathrm{Cr}$ neutrino source experiment with the GALLEX detector, Phys. Lett. B 342 (1995) 440 [INSPIRE].

[60] GALLEX collaboration, Final results of the ${ }^{51} \mathrm{Cr}$ neutrino source experiments in GALLEX, Phys. Lett. B 420 (1998) 114 [INSPIRE].

[61] F. Kaether, W. Hampel, G. Heusser, J. Kiko and T. Kirsten, Reanalysis of the GALLEX solar neutrino flux and source experiments, Phys. Lett. B 685 (2010) 47 [arXiv:1001.2731] [INSPIRE]. 
[62] D. Abdurashitov et al., The Russian-American gallium experiment (SAGE) Cr neutrino source measurement, Phys. Rev. Lett. 77 (1996) 4708 [INSPIRE].

[63] SAGE collaboration, Measurement of the response of the Russian-American gallium experiment to neutrinos from a ${ }^{51} \mathrm{Cr}$ source, Phys. Rev. C 59 (1999) 2246 [hep-ph/9803418] [INSPIRE].

[64] SAGE collaboration, Measurement of the solar neutrino capture rate with gallium metal. III: Results for the 2002-2007 data-taking period, Phys. Rev. C 80 (2009) 015807 [arXiv:0901.2200] [INSPIRE].

[65] D. Krofcheck et al., Gamow-Teller strength function in ${ }^{71}$ Ge via the $(p, n)$ reaction at medium-energies, Phys. Rev. Lett. 55 (1985) 1051 [inSPIRE]. 